\title{
Improvised leach protocol for significant reduction in power in wireless sensor networks
}

\author{
Shaik Karimullah \\ Assistant Professor, Department of ECE, Annamacharya Institute of Technology and Sciences, Rajampet, \\ Andhra Pradesh, India \\ munnu483@gmail.com
}

Received: $14^{\text {th }}$ September 2020, Accepted: $26^{\text {th }}$ November 2020, Published: $31^{\text {st }}$ December 2020

\begin{abstract}
The wireless sensor network's energy consumption is largely influenced by the architecture of clustered hierarchical routing algorithms (WSN). When it comes to WSNs, an application-specific protocol called LEACH has been developed as a low-energy clustering hierarchy. If you don't know how cluster heads (CH's) rotate, then the LEACH protocol will increase the energy consumption of the network. The energy efficiency of a WSN can be improved with a new, modified routing protocol, which we describe in this work. In this research, we propose a new, energy-efficient routing technique for WSNs. Net energy and average network energy are considered in the new enhanced energy-efficient LEACH protocol (IEE-LEACH). It is proposed that IEELEACH take into account the optimal number of $\mathrm{CHs}$ and forbids the building of nodes clusters closer to the base station in order to achieve adequate efficiency in reducing sensor energy consumption (BS). It is suggested that the proposed IEE-LEACH uses a new threshold for selecting CHs from the sensor nodes in order to improve network energy efficiency even more. Simulation results reveal that the proposed routing protocol greatly reduces the energy consumption of WSNs compared to the current routing protocol.
\end{abstract}

Keywords: WSN;TDMA;LEACH-I;NODE DEGREE; EFFICIENT ENERGY

\section{Introduction}

Networks with thousands of nodes that can sense, communicate with one another, and perform computational and processing tasks are known as Wireless Sensor Networks (WSNs). When developing life-critical protocols, one of the most important considerations is how to conserve energy. Batteries in sensor nodes are tiny and have a limited capacity. They can either be deployed manually or automatically deleted. Clusters are an efficient and scalable way to manage a wireless network. As a result, the energy consumption per cluster member is larger. Using a probabilistic technique to tackle the energy clustering problem, LEACH is the most often used clustering approach. One goal of this study is finding a solution to the energy problem and devising a method for picking the cluster head in such a way as to cut down on electricity use as much as possible. Aside from military applications, sensor networks have also been utilised for environmental monitoring, safety monitoring, and other purposes. The architecture of a sensor network is driven by a variety of considerations, such as faulttolerant communication, scalability, power consumption, data delivery methods, data aggregation/fusion, and node deployment. The LEACH network protocol uses hierarchical routing of wireless sensors to extend the network's service life. Nodes other than the cluster's central node send information to the cluster's central node. In the event of a cluster-head node's death, all nodes that cluster with the base station lose the ability to communicate. LEACH is a network technology that uses wireless sensor hierarchy routing to increase the service life of the network. Data from non-cluster-head nodes should be sent to the cluster-head nodes. So when a cluster-head node dies, all nodes in the cluster. Communication between the cluster and the base station is disrupted. 


\section{Literature survey}

In "A forward-aware factor-based energy-balanced routing approach for wireless sensor networks," Zhang, D.;Li, G.; Zheng, K.; Ming, X.; Pan, Z. suggested "A forward-aware factor-based energy balancing." This provides data for the collection and transmission of various environmental characteristics to the base station (BS).

"Routing vector protocol accuracy as a network cycle property" was proposed by Sobrinho, J.L. To track and detect surveillance applications such military forest fire detection, human health detection, etc., a "new method for constructing autonomous wireless sensor nodes based on RF energy harvesting devices" was proposed in IEEE Trans. As a result, researchers have gained attention in recent years. It's been a long time since we've seen the likes of Li XF and Wang HB as well as Xu LZ, Song J, and Yang S.X. in the same paper. They proposed "A novel evolution-based routing algorithm for environmental monitoring wireless sensor networks" in a paper coauthored by Jianping Shen, Xiaoyu Wang, Cheng-Chuan Lai, and Peter C.K. Hung. The batteries in WSNs are difficult to replace or recharge because they are often used in hazardous environments. The manual maintenance of the network is extremely time consuming, which raises some concerns about the use of WSNs.

Sohn, I.; Lee, J.; and S.H. 'Affinity propagation-based clustering hierarchy for wireless sensor networks' has been proposed. As a means of addressing these issues, protocols and hardware architectures should prioritise optimal use of SN battery resources. Zhang; $\mathrm{Xu}$; Hui; $\mathrm{Hu}$; L. To reduce power consumption while still maintaining high performance, the authors (Heinzelman, Chandrakasan, and Balakrishnan) came up with an AGNES-based energy-efficient wireless sensor routing protocol. An energy-efficient communication protocol has been devised for wireless microsensor networks. A number of new routing algorithms have been presented during the 33rd Annual Hawaii International Conference on System Sciences (HICSS), which took place in the Hawaiian island of Maui, U.S.A.

N.R. Roy; recommended "P. Chandra Observation concerning LEACH's optimal cluster estimate." The development of clusters and the various modalities of data transfer connectivity were the most frequently cited strategies. In contrast to non-clustering protocols, cluster-based routing techniques may effectively utilise the network's SNs. Hosen, A.; Cho, G.; and others As suggested by T.Kaur, D.Kumar, and others. a cluster-based routing strategy for wireless sensor networks that prioritises energy. Wireless sensor networks use a particle swarm-based clustering technique that is uneven and fault-tolerant." The cluster head $(\mathrm{CH})$ is the person in charge of ensuring that the associated data is kept to a minimum so that the system's ultimate volume is kept to a minimum. In addition, the $\mathrm{CH}$ will provide the aggregated data to the BS.

Behera et al have proposed that there are several clusters of nodes in cluster-based routing protocols to reduce energy consumption for long-distance communication. As a result of the large energy imbalance between CHs and other nodes, clustering will reduce overall energy consumption and balance node burden. Energy-efficient clustering is another way to increase network resilience and reduce energy consumption. For this reason, most clustering methods make use of optimal $\mathrm{CH}$ selection in order to keep the SNs alive for as long as possible. Proposed Wireless Sensor Network Principles by Misra and Obaidat of Cambridge University shows us that SNs waste more energy when communicating than when processing.

Ishmael Tarhani, J. Tarhani, M. Tarhani, M. Tarhani and Y.S. Kavian are the authors of this study. The study was published in the Journal of Biological Chemistry. A new wireless sensor network protocol called the 'Enhanced message-passing LEACH protocol' has been proposed. A different protocol uses multi-hop communication, which leads to excessive overhead transmission at nodes near the BS and, as a result, holes in the sensor field.

In their paper, "Wireless microsensor networks adopt a common application protocol architecture," A.P. Chandrakasan and H. Balakrishnan posited this idea: Clustering methods have been specifically recommended for WSNs in order to address the energy hole problem and extend the network's lifespan. Comparing common routing methods, the Low Energy Adaptive Clustering Hierarchy (LEACH) is the most efficient hierarchical routing technique in terms of saving energy. 
An application protocol architecture for wireless microsensor networks was proposed by A.P. Chandrakasan and $\mathrm{H}$. Balakrishnan. It is the responsibility of $\mathrm{CH}$ to receive, aggregate, compress, and distribute the collected data in this protocol. Each cluster in the network selects a node in this protocol in a probabilistic manner. Using the LEACH routing protocol, each cluster is assigned a single $\mathrm{CH}$ node, which is then rotated. This reduces the WSN's energy consumption. LEACH has been thoroughly researched, but there are still several issues that need to be addressed because $\mathrm{CH}$ selection is based on random round-robin, the number of $\mathrm{CHs}$ is arbitrarily in-round and nodes at network boundaries are elected as $\mathrm{CHs}$. Those variables aren't taken into account while calculating the final $\mathrm{CH}$ distribution, threshold status, or residual energy across all nodes. As a result of these issues, power costs would rise.

It was suggested by Singh, S.K and Kumar P; and Singh, J.P; Successors to the LEACH Protocol: A Research Report LEACH-XMP (LEACH Extended Message Passing) has been presented as an energy consumptionminimizing method for $\mathrm{CH}$ nodes that avoids oversimplification. During the first cycle, these protocols are primarily concerned with distributing the CH's energy usage among its assistants. The network's lifespan would be reduced as a result of this distribution. As a way to overcome the shortcomings of conventional approaches and further increase the lifespan of WSNs, we propose a new, more energy-efficient LEACH routing protocol (IEE-LEACH).

\section{Research Methodology}

The proposed protocol has four parameters for threshold setting: beginning node energy, residual node energy, total network energy, and average network energy. These micro-sensors can sense, communicate, and perform computation and processing tasks in a Wireless Sensor Network (WSN). For many applications that do not require human intervention, low-power, low-cost, and rapid deployment can be huge advantages. When developing life-critical protocols, one of the most important considerations is how to conserve energy. Batteries for sensor nodes are compact and power-limited. Automatically or manually, they can be dropped at any time. You can effectively manage the wireless network if you take advantage of the clusters. Sensor networks were first employed primarily for military purposes, but they have since been utilised for a variety of other purposes, including environmental monitoring, health monitoring, and more. Fault-tolerance communication, scalability and power consumption are just a few of the considerations that go into the design of a sensor network. In this case, a TDMA schedule is established for each node by the cluster head, who is aware of all member nodes and so knows exactly when to broadcast their data.

\section{Results and Discussion}

Based on network creation factors such as the number of nodes, sinks, and various energy values depending on clusters, the Leach protocol is tested in this study. Figure 1 depicts the groupings of cells shown here.

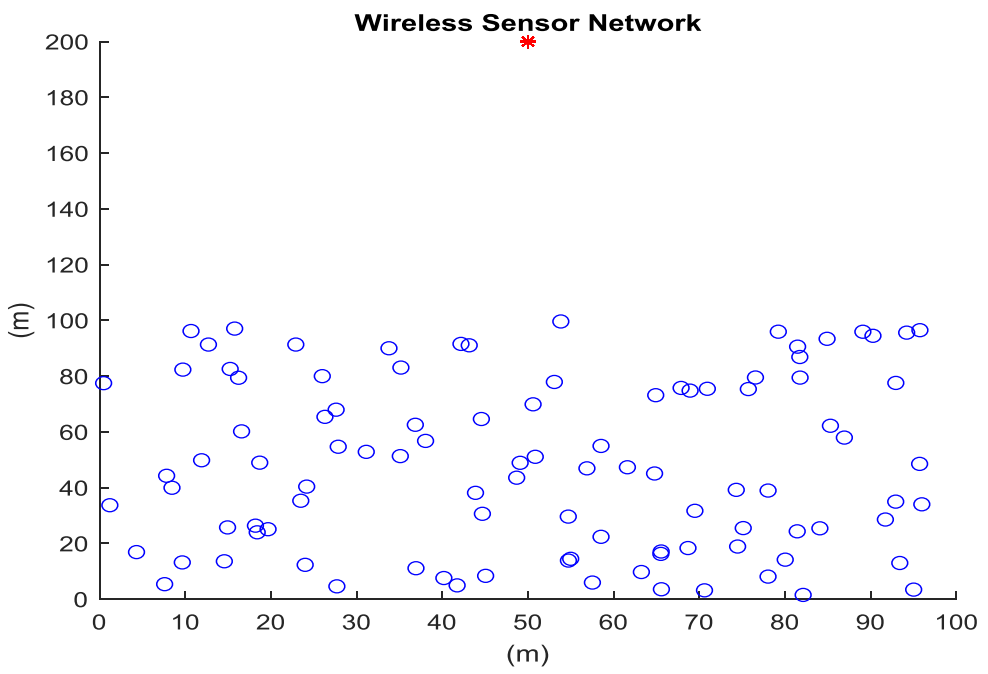

Fig 1: Wireless sensor network 
As a result, wireless sensor networks are created. The set-up phase is then started, which shows the operational nodes each round in figure 2 and operating nodes per transmission in figure 3.

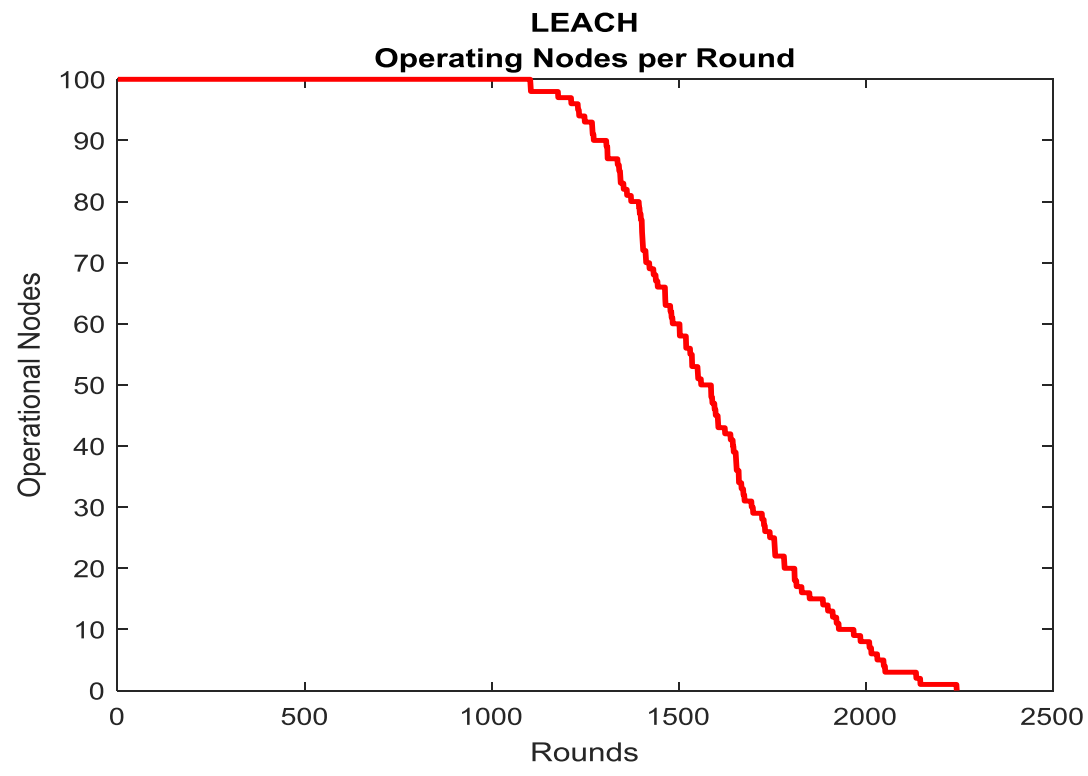

Fig 2: Operating nodes per round

LEACH

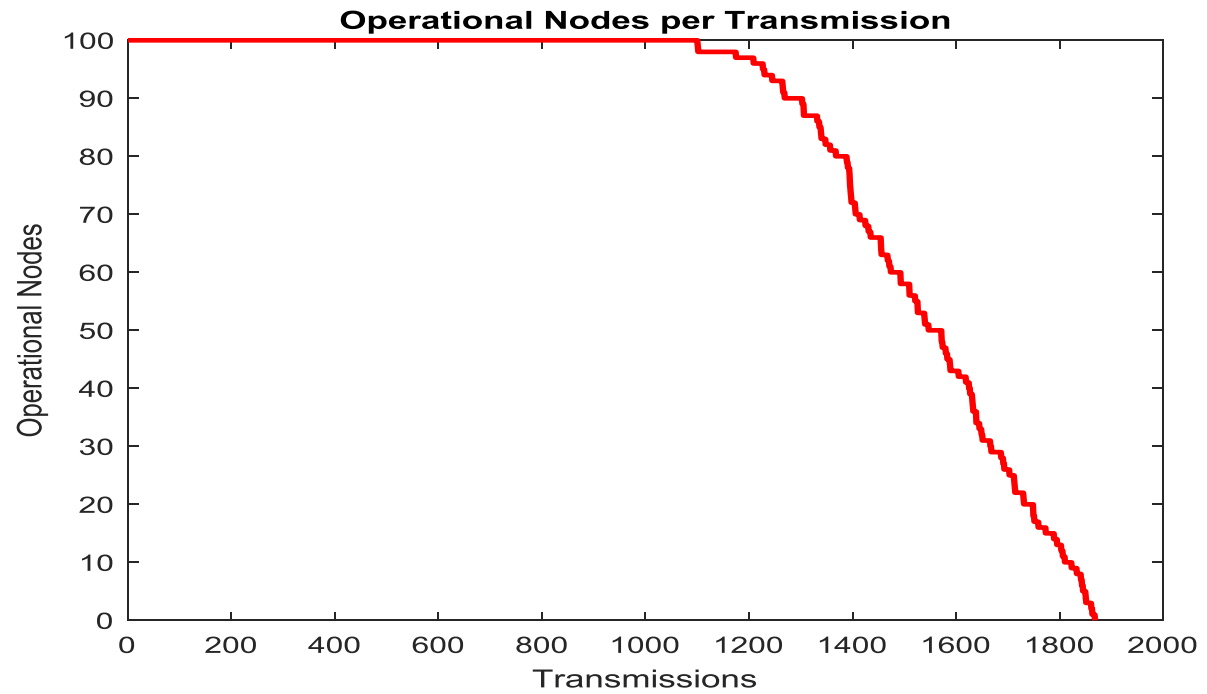

Fig 3: Operating nodes per transmission

It is clear from these two graphs that the number of operational nodes reduces as the number of nodes and transmission diminishes. 


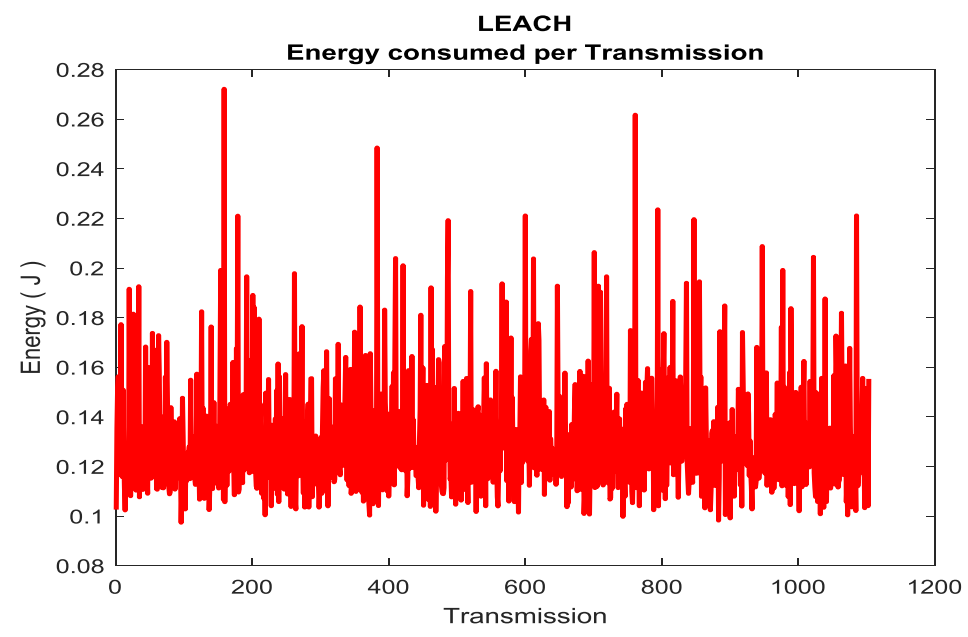

Fig 4: Consumed energy per transmission

After the selection of a cluster head, the steady phase state is commenced, which indicates the energy consumption per transmission as shown in the above figure and also the average energy consumed by a node per Transmission is also verified in the below figure.

LEACH

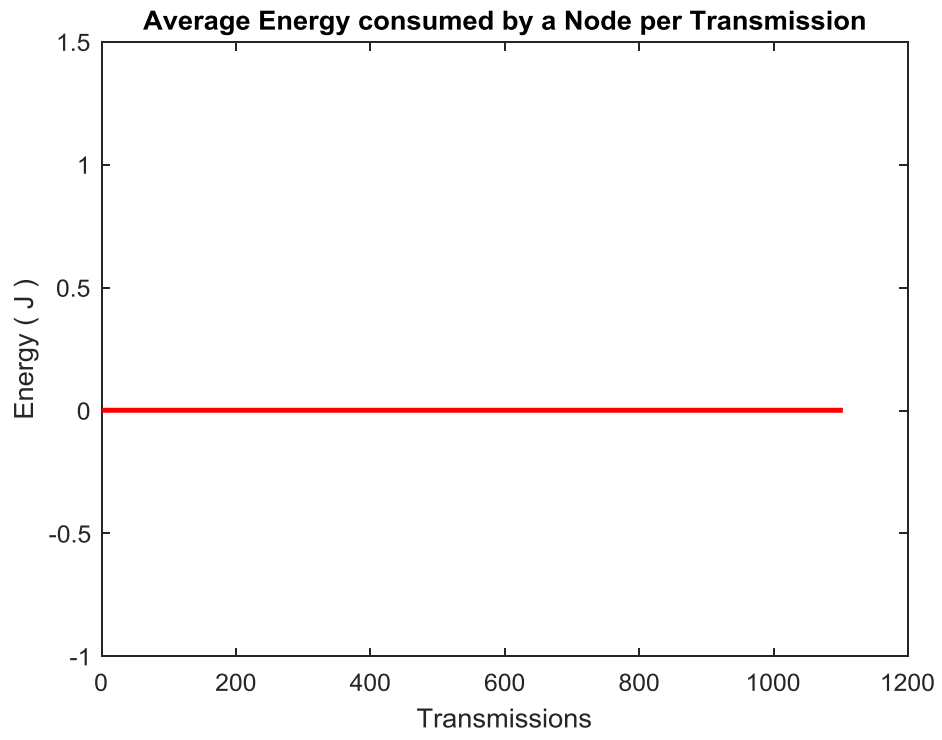

Fig 5: Average energy consumed by a node per transmission

It has been found that when the number of nodes increases, energy usage climbs gradually rather than abruptly as depicted by following Table 1 .

Table 1: Energy consumption for different nodes

\begin{tabular}{|l|l|}
\hline Number of Nodes & Energy consumed(micro Watt) \\
\hline 100 & 0.31 \\
\hline 96 & 0.29 \\
\hline 89 & 0.248 \\
\hline 84 & 0.226 \\
\hline 80 & 0.216 \\
\hline 74 & 0.22 \\
\hline
\end{tabular}




\section{Conclusion}

It was the goal of this article to examine how the battery's power consumption has been affected and decreased as a result of normal nodes selecting suitable cluster heads that are located at a minimum distance from the base station. A simulation-based efficiency analysis was carried out to compare the power consumption of the proposed approach strategy to LEACH. Power consumption is reduced and its life span is prolonged as a result of the findings. As the number of rounds is raised, so will the lowered power need. It's clear that the new method outperforms LEACH.

\section{References}

1. Bahbahani, M.S.; Alsusa, E. A cooperative clustering protocol with duty cycling for energy harvesting enabled wireless sensor networks. IEEE Trans. Wirel. Commun. 2018, 17, 101-111. [CrossRef]

2. Behera, T.M.; Samal, U.C.; Mohapatra, S.K. Energy-efficient modified LEACH protocol for IoT application.IET Wirel. Sens. Syst. 2018, 8, 223-228. [CrossRef]

3. Hosen, A.; Cho, G. An energy centric cluster-based routing protocol for wireless sensor networks. Sensors 2018, 18, 1520. [CrossRef] [PubMed]

4. Kaur, T.; Kumar, D. Particle swarm optimization-based unequal and fault tolerant clustering protocol for wireless sensor networks. IEEE Sens. J. 2018, 18, 4614-4622. [CrossRef]

5. Mouapi, A.; Hakem, N. A new approach to design autonomous wireless sensor node based on RF energy harvesting system.. [CrossRef] [PubMed]

6. Obaidat, M.S.; Misra, S. Principles of Wireless Sensor Networks; Cambridge Univ. Press: Cambridge, UK, 2014

7. Li, X.F.; Xu, L.Z.; Wang, H.B.; Song, J.; Yang, S.XSensors 2010, 10, 5425-5442. [CrossRef] [PubMed]

8. Ma, T.; Hempel, M.; Peng, D.; Sharif, H. A survey of energy-efficient compression and communication techniques for multimedia in resource constrained systems. IEEE Commun. Surv. Tutor. 2013, 15, 963-972. [CrossRef]

9. Roy, N.R.; Chandra, P. A note on optimum cluster estimation in LEACH protocol. IEEE Access 2018, 6, 65690-65696. [CrossRef]

10. Shaik Karimullah, Dr. D.Vishnuvardhan ,"Experimental Analysis Of Optimization Techniques For Placement And Routing In Asic Design” ICDSMLA 2019, Lecture Notes in Electrical Engineering 601, Springer Nature Singapore Pte Ltd. 2020.

11. Shaik Karimullah and D.Vishnuvardhan, "Iterative Analysis of Optimization Algorithms for Placement and Routing in Asic Design”, ICDSMLA 2019, Lecture Notes in Electrical Engineering 601, Springer Nature Singapore Pte Ltd. 2020.

12. Sharma, D.; Bhondekar, A.P. Traffic and energy aware routing for heterogeneous wireless sensor networks.IEEE Commun. Lett. 2018, 22, 1608-1611. [CrossRef]

13. Zhang, Y.; Liu, M.; Liu, Q. An energy-balanced clustering protocol based on an improved CFSFDP algorithm for wireless sensor networks. Sensors 2018, 18, 881. [CrossRef] [PubMed]

14. Zhao, Z.; Xu, K.; Hui, G.; Hu, L. An energy-efficient clustering routing protocol for wireless sensor setworks based on AGNES with balanced energy consumption optimization. Sensors 2018, 18, 3938. [CrossRef] 